\title{
Impact of Primary Network on Secondary Network with Generalized Selection Combining
}

\author{
Yansha Deng, Student Member, IEEE, Maged Elkashlan, Member, IEEE, Nan Yang, Member, IEEE, \\ Phee Lep Yeoh, Member, IEEE, and Ranjan K. Mallik, Fellow, IEEE
}

\begin{abstract}
We propose cognitive spectrum sharing with generalized selection combining (GSC) at the secondary user (SU) in the presence of multiple primary transceivers with outdated channel information. Our main motivation is to determine the impact of GSC and outdated channel information on the outage probabiliy of cognitive spectrum sharing subject to two practical power constraints: 1) maximum transmit power at the $S U$ transmitter, and 2) peak interference temperature at the PU receiver. We derive new closed-form expressions for the exact and asymptotic outage probability in Rayleigh fading. Our expressions provide concise representations of the diversity order and the array gain. We confirm that the diversity order of GSC is entirely dependent on the secondary network and is equal to the available number of receive antennas at the SU. This result is consistent with those of maximal-ratio combining (MRC) and selection combining (SC) in cognitive spectrum sharing. More importantly, our results show that the outage probability decreases with increasing the correlation coefficient of the outdated channel.
\end{abstract}

Index Terms-Spectrum sharing, diversity combining, generalized selection combining, outdated channel information.

\section{INTRODUCTION}

Cognitive radio has been proposed as a promising means to cope with the rapid growth of volume-intensive bandwidthhungry mobile applications such as online gaming, social networking, and high-definition (HD) video streaming. The socalled underlay cognitive radio allows the secondary user (SU) and the primary user (PU) to transmit concurrently in the same spectrum provided that the interference from the SU to the PU does not exceed a predetermined interference threshold [1,2]. The challenge is to effectively coordinate the transmit power at the SU transmitter (SU-Tx), the interference power at the PU receiver (PU-Rx) from the SU-Tx, and the interference power at the SU receiver (SU-Rx) from the PU transmitter (PU-Tx). While cognitive radio increases the reliability of the secondary network, interference requirements in the primary network are often contradictory [3]. The goal of this paper

Copyright (c) 2013 IEEE. Personal use of this material is permitted. However, permission to use this material for any other purposes must be obtained from the IEEE by sending a request to pubs-permissions@ieee.org.

Y. Deng and M. Elkashlan are with the School of Electronic Engineering and Computer Science, Queen Mary, University of London, London E1 4NS, UK (email: \{yansha.deng, maged.elkashlan\}@eecs.qmul.ac.uk).

N. Yang is with the Research School of Engineering, Australian National University, Canberra, Australia (email: nan.yang@unsw.edu.au).

P. L. Yeoh is with the Department of Electrical and Electronic Engineering, University of Melbourne, Parkville, Vic. 3010, Australia (email: phee.yeoh@unimelb.edu.au).

R. K. Mallik is with the Department of Electrical Engineering, Indian Institute of Technology - Delhi, Hauz Khas, New Delhi 110016, India (email: rkmallik@ee.iitd.ernet.in). is therefore to address this delicate balance by proposing generalized selection combining (GSC) at the SU-Rx.

To maximize the link reliability of the secondary network and minimize the interference at the primary network, receive diversity at the SU has been applied in interference-limited spectrum sharing networks. While the outage probability and the bit error rate of cognitive networks with a single antenna at the SUs and the PUs were derived in $[4,5]$, the capacity of the more general case of multiple antennas at the SU was considered in $[6,7]$. In [6,8], maximal-ratio combining (MRC) was applied at the SU. It was shown in [8] that the capacity of cognitive radio with average interference power constraint increases with MRC. In [6], the impact of peak interference power constraint on the capacity was characterized. Considering GSC at the SU, [7] evaluated the capacity with peak interference power constraint. Apart from capacity, the outage probability is another important metric to comprehend the fundamental limits of cognitive radio $[9,10]$. The practical consideration of interference from PU-Tx to SU$\mathrm{Rx}$ was recently examined in [11]. However, references [4-11] have all assumed that the SU has full channel-state information (CSI) between the SU-Tx and PU-Rxs, and the impact of GSC in the presence of multiple primary transceivers is less well understood.

In this paper, we view cognitive radio from the viewpoint of GSC as a low power design in interference-limited spectrum sharing networks under outdated CSI between the SU-Tx and PU-Rxs. We consider a general scenario where the SU transmits in the presence of $M$ PU-Txs and $M$ PU-Rxs. The main objective is to enhance the transmission reliability of the secondary network while strictly meet the interference constraint at the primary network, which can be achieved by varying the number of combined antennas at the SU-Rx using GSC. Compared to other diversity combining techniques, GSC is also capable to combat channel estimation errors by eliminating weaker SIRs [12]. To answer some of the pressing questions that face spectrum sharing networks, we relate the maximum transmit power at the SU-Tx with the peak interference power at the PU-Rxs and the interference power from the PU-Txs. Different from previous studies, with GSC, the SU-Rx is allowed to select a subset of its receive antennas so as to balance the transmit power at SU-Tx and the interference power at PU-Rx in the presence of multiple PU transceivers. By doing so, less transmit power at the SUTx is required to achieve the same outage performance, which in turn reduces the interference at the PU-Rxs. Additionally, a flexible back-off power control mechanism is adopted to guarantee the quality of service $(\mathrm{QoS})$ of the primary network. 
Owing to this fact, our outcomes are presented in the form of new expressions for the exact and the asymptotic outage probability. Our results bridge the gap between MRC and selection combining (SC) by allowing the SU-Rx to combine the $L_{c}$ strongest out of its $L$ available receive antennas. We show that the full diversity order is achieved. The diversity order is entirely determined by the secondary network and is equal to $L$. We also present the performance gap between GSC, MRC, and SC as a simple ratio of their respective array gains. An interesting conclusion is reached that $M$ imposes a negative impact on the outage probability, while $L_{c}$ has a positive impact on the outage probability. Furthermore, the outage probability decreases with increasing correlation coefficient of the outdated CSI channel.

\section{Network And Channel Description}

We consider a realistic underlay spectrum sharing network with one pair of SU-transceiver and $M$ pairs of PUtransceivers. In the secondary network, we assume a receiver diversity where the SU-Rx is equipped with $L$ receive antennas. The $L_{c}$ receive antennas are selected based on the channel from the SU-Tx to the SU-Rx. All other terminals are equipped with only one antenna. We assume that only partial channel knowledge of the interference channel from the SU-Tx to the PU-Rxs is available at the SU-Tx. The CSI of $h_{1 m}$ provided to the SU-Tx is outdated due to the time-varying nature of the wireless link [13]. We describe the outdated CSI of the SU-Tx to the $m$ th PU-Rx channel using the correlation model $[14,15]$ as

$$
h_{1 m}=\rho \hat{h}_{1 m}+\sqrt{1-\rho^{2}} \varepsilon,
$$

where $m \in\{1, \ldots, M\}, \hat{h}_{1 m}$ is the outdated channel information available at the SU-Tx, $\varepsilon$ is a complex Gaussian random variable with zero mean and unit variance, and uncorrelated with $h_{1 m}$. The correlation coefficient $\rho$ is a constant, which is used to evaluate the impact of channel estimation error, mobility, and feedback delay on the CSI [15]. Note that we assume that both the outdated channel information $\hat{h}_{1 m}$ and the correlaction coefficient $\rho$ are available at SU-Tx.

We assume that both the secondary channel and the primary channel are subject to quasi-static fading where the channel coefficients are constant for each transmission block but vary independently between different blocks. The primary and secondary networks are subject to independent and identically distributed (i.i.d.) Rayleigh fading. The estimated channel power gain from the SU-Tx to the $m$ th PU-Rx channel is an exponentially distributed RV with parameter $\frac{1}{\hat{\alpha}_{1}}$. The instantaneous channel power gains from the SU-Tx to the $m$ th PU-Rx channel, from the $m$ th PU-Tx to the $l$ th transmit antenna at SU-Rx, and from the SU-Tx to the $l$ th receive antenna at SU-Rx are exponentially distributed RVs with parameters $\frac{1}{\alpha_{1}}, \frac{1}{\alpha_{2}}$, and $\frac{1}{\alpha_{3}}$, respectively, where $l \in\{1, \ldots, L\}$ and $\alpha_{1}=\rho^{2} \hat{\alpha}_{1}+\left(1-\rho^{2}\right)$. Note that here we mainly focus on the outdated channel effect and the impact of GSC, thus, the different path-loss and shadowing effect between the SUTx and PU-Rxs links resulting from the geometry are not taken into consideration in this work. This assumption is also applicable to the scenario where PU-Rxs are located in a compact and small area.

\section{A. Interference Outage Probability}

According to underlay spectrum sharing, the interference from the secondary network impinged on the primary network should remain below a pre-defined peak interference temperature $Q$ [16]. When only the outdated interference channel information is available at SU-Tx, this strict interference requirement can not be satisfied at all times. Therefore, the PURxs should tolerate outages occuring for a certain percentage of time, which is named as the interference outage. In [15], it was been proved that the interference outage probability is always 0.5 as long as the maximum transmission power is ignored. Therefore, instead of the strict peak interference constraint, a more flexible constraint known as the power margin, which is based on the interference outage probability is considered to prevent the primary transmission from degradation. The transmit power and the interference outage probability are denoted as

$$
P\left(\rho,\left|\hat{h}_{1 m^{*}}\right|^{2}\right)=\min \left(P_{T}, k_{I} \frac{Q}{\left|\hat{h}_{1 m^{*}}\right|^{2}}\right)
$$

and

$$
P_{o}=1-\operatorname{Pr}\left\{\min \left(P_{T}, k_{I} \frac{Q}{\left|\hat{h}_{1 m^{*}}\right|^{2}}\right)\left|h_{1 m^{*}}\right|^{2} \leq Q\right\},
$$

respectively, where $P_{\mathrm{T}}$ is the maximum available transmit power at the SU-Tx, and $\left|\hat{h}_{1 m^{*}}\right|^{2}$ is the largest estimated channel power gain from the SU-Tx to the $M$ PU-Rxs available at SUTx. We assume the power margin factor $k_{I}=1$ for the perfect CSI between SU-Tx and PU-Rx link. Since the closed-form expression for the power margin factor $k_{I}$ is intractable [14], therein, we numerically evaluate $k_{I}$ based on (3).

\section{Signal-to-Interference RATiO}

In the secondary network, GSC is applied to combine the $L_{c}\left(1 \leq L_{c} \leq L\right)$ antennas with the strongest received signals from $L$ available receive antennas at the SU-Rx. The channel coefficients from the SU-Tx to the $l$ th receive antenna at the SU-Rx are denoted as $g_{l}(1 \leq l \leq L)$. Let $\left|g_{1}\right|^{2} \geq\left|g_{2}\right|^{2} \geq$ $\cdots\left|g_{L}\right|^{2} \geq 0$ be the order statistics obtained by sorting $\left\{g_{l}\right\}_{l=1}^{L}$ in decreasing order of magnitude [7]. After performing GSC [17], the received signal at the SU-Rx is given by

$$
y=\frac{\boldsymbol{G}^{H}}{\|\boldsymbol{G}\|} \boldsymbol{G} x_{T}+\underbrace{\sum_{m=1}^{M} \frac{\boldsymbol{G}^{H}}{\|\boldsymbol{G}\|} \boldsymbol{H}_{2 m} x_{m}}_{\text {Interferences from PU - Txs }}+\frac{\boldsymbol{G}^{H}}{\|\boldsymbol{G}\|} \boldsymbol{n}
$$

where $x_{T}$ is the transmit signal at the SU-Tx, $\boldsymbol{G}=$ $\left[g_{1}, g_{2}, \ldots, g_{L_{c}}\right]^{T}$ is the selected channel vector at the SU$\mathrm{Rx}, x_{m}$ is the transmit signal at the $m$ th PU-Tx, $\boldsymbol{H}_{2 m}$ is the $L_{c} \times 1$ channel vector from the $m$ th PU-Tx to $L_{c}$ antennas with strongest receive signals at the SU-Rx, and $\boldsymbol{n} \sim \mathcal{C N}\left(\mathbf{0}, \sigma^{2} \mathbf{I}_{L_{c}}\right)$ is the $L_{c} \times 1$ additive white Gaussian noise (AWGN) vector. Similar to some practical applications such as cellular CDMA networks and dense ad hoc networks 
[18], we consider the interference-limited scenario of cognitive networks with multiple primary transceivers where the noise is typically negligible compared to the sum of interferences from the multiple PU-Txs. As such, the SIR can be used interchangeably with SINR without much appreciable loss of accuracy [19-21]. Based on (4), the instantaneous received signal-to-interference ratio (SIR) is given by

$$
\tilde{\gamma}=\min \left(P_{\mathrm{T}}, \frac{k_{I} Q}{Y}\right) \frac{X}{V},
$$

where $X=\|\boldsymbol{G}\|^{2}, Y=\left|\hat{h}_{1 m^{*}}\right|^{2}, V=\sum_{m=1}^{M} P_{\mathrm{I}}\left|\frac{\boldsymbol{G}^{H} \boldsymbol{H}_{2 m}}{\|\boldsymbol{G}\|}\right|^{2}$ is the interference power from the PU-Txs to the SU-Rx.

\section{OUtage Probability}

\section{A. Exact Analysis}

Outage probability is an important metric to characterize the performance of cognitive networks in practice. In cognitive networks, an outage occurs if the instantaneous received SIR at the SU drops below a given threshold $\gamma_{\mathrm{th}}$. Based on this, the outage probability is formulated as

$$
P_{\text {out }}=\operatorname{Pr}\left\{\tilde{\gamma} \leq \gamma_{\text {th }}\right\}=F_{\tilde{\gamma}}\left(\gamma_{\text {th }}\right),
$$

where $\operatorname{Pr}\{\cdot\}$ denotes the probability and $F_{\tilde{\gamma}}\left(\gamma_{\text {th }}\right)$ denotes the cumulative distribution function (CDF) of $\tilde{\gamma}$. To derive $F_{\tilde{\gamma}}\left(\gamma_{\mathrm{th}}\right)$, we first present the statistics of the channel power gain $\|\boldsymbol{G}\|^{2}$ as follows.

The CDF of $\|\boldsymbol{G}\|^{2}$ is obtained using [17, eq. (4)] and the multinomial expansion [22] which results in

$$
F_{\|\boldsymbol{G}\|^{2}}(x)=\sum_{S_{k} \in S_{K}} \alpha_{k} x^{\beta_{k}} e^{-\delta_{k} \frac{x}{\alpha_{3}}},
$$

where $S_{K}=\left\{S_{k} \mid \sum_{l=0}^{L} m_{k, l}=1\right\}$ with $\left\{m_{k, l}\right\} \in Z^{+}$. We note that $S_{K}$ refers to all possible combinations of $\left\{m_{k, l}, l=0,1, \ldots, L\right\}$ which satisfy $\sum_{l=0}^{L} m_{k, l}=1$. The parameters in (7) are defined as

$$
\begin{gathered}
\alpha_{k}=\left(\varepsilon_{0}\right)^{m_{h, 0}} \prod_{l=1}^{L_{c}}\left(\frac{\varepsilon_{l}}{\Gamma(l)}\right)^{m_{h, l}} \prod_{l=L_{c}+1}^{L} \varepsilon_{l}^{m_{h, l},} \\
\beta_{k}=\sum_{l=1}^{L_{c}}(l-1) m_{k, l},
\end{gathered}
$$

and

$$
\delta_{k}=\sum_{l=1}^{L_{c}} m_{k, l}+\sum_{l=L_{c}+1}^{L} \frac{l}{L_{c}} m_{k, l},
$$

where $\varepsilon_{l}$ is given by

$$
\begin{aligned}
& \varepsilon_{l}=
\end{aligned}
$$

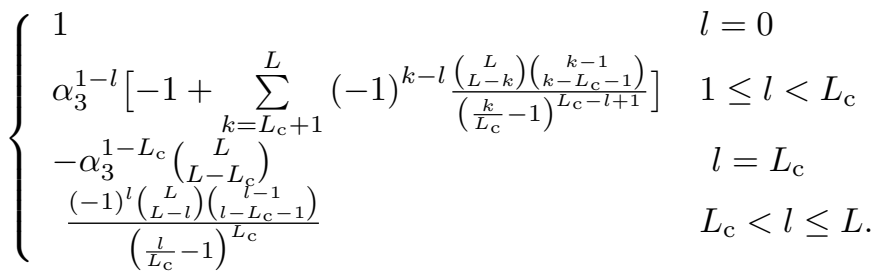

The CDF of $\left|\hat{h}_{1 m^{*}}\right|^{2}$ is given by

$$
F_{\left|\hat{h}_{1 m^{*}}\right|^{2}}(x)=\left(1-e^{-\frac{x}{\alpha_{1}}}\right)^{M} .
$$

Recall that we define $V=\sum_{m=1}^{M} P_{\mathrm{I}}\left|\frac{\mathbf{G}^{H} \mathbf{H}_{2 m}}{\|\mathbf{G}\|}\right|^{2}$. According to [23], $\frac{\mathbf{G}^{H} \mathbf{H}_{2 m}}{\|\mathbf{G}\|}$ is a zero-mean complex Gaussian vector, which is independent of $\mathbf{G}^{H}$ and $\|\mathbf{G}\|$, and $\left|\frac{\mathbf{G}^{H} \mathbf{H}_{2 m}}{\|\mathbf{G}\|}\right|^{2}$ follows the chi-square distribution with 2 degrees of freedom. As such, the probability distribution function (PDF) of $V$ is given by

$$
f_{V}(x)=\left(\frac{1}{P_{I} \alpha_{2}}\right)^{M} \frac{x^{M-1} e^{-\frac{x}{P_{I} \alpha_{2}}}}{\Gamma(M)} .
$$

Based on the CDF and PDF of $X, Y$, and $V$, we present a closed-form expression for the outage probability in the following theorem.

Theorem 1. The closed-form expression for the outage probability of spectrum sharing networks with GSC and outdated CSI between SU-Tx and PU-Rxs is derived as

$$
P_{\text {out }}\left(\gamma_{t h}\right)=\frac{\Gamma\left(\beta_{k}+M\right)}{\Gamma(M)} \sum_{S_{k} \in S_{K}} \alpha_{k} \gamma_{t h}^{\beta_{k}} \Xi\left(\gamma_{t h}\right),
$$

where

$$
\begin{aligned}
& \Xi\left(\gamma_{t h}\right)=\left(1-e^{-\frac{k_{I} Q}{\alpha_{1} P_{T}}}\right)^{M}\left(\frac{1}{P_{I} \alpha_{2}}\right)^{M}\left(\frac{1}{P_{T}}\right)^{\beta_{k}} \\
& \frac{1}{\left(\frac{\delta_{k} \gamma_{t h}}{P_{T} \alpha_{3}}+\frac{1}{P_{I} \alpha_{2}}\right)^{\beta_{k}+M}}+\sum_{m=1}^{M}\left(\begin{array}{c}
M \\
m
\end{array}\right)(-1)^{m+1}\left(\frac{\alpha_{3}}{\hat{\alpha}_{1} \delta_{k} \gamma_{t h}}\right)^{\beta_{k}+M} \\
& \left(\frac{k_{I} Q}{P_{I} \alpha_{2}}\right)^{M} \beta_{k} ! e^{-\frac{m k_{I} Q}{\hat{\alpha}_{1} P_{T}}} \sum_{i=0}^{\beta_{k}} \frac{\left(\frac{k_{I} Q}{P_{T}}\right)^{i}}{i !}\left(\frac{m}{\hat{\alpha}_{1}}\right)^{i+M} \\
& \Phi\left(\beta_{k}+M, M+i ;\left(\frac{1}{P_{I} \alpha_{2}}+\frac{\delta_{k} \gamma_{t h}}{\alpha_{3} P_{T}}\right) \frac{\alpha_{3} m k_{I} Q}{\hat{\alpha}_{1} \delta_{k} \gamma_{t h}}\right)
\end{aligned}
$$

where $\Gamma(\cdot)$ is the gamma function [24, Eq. (8.310.1)], and $\Phi(\cdot, \cdot ; \cdot)$ is the confluent hypergeometric function [24, Eq. (9.211.4)].

Proof. See Appendix A.

Lemma 1. The outage probability of spectrum sharing networks with perfect CSI, and only one pair of primary transceiver and secondary transceiver with single antenna is derived as

$$
\begin{aligned}
P_{\text {out }}\left(\gamma_{t h}\right)= & 1-\frac{\left(1-e^{-\frac{Q}{\hat{\alpha}_{1} P_{T}}}\right)}{\left(\frac{P_{I} \alpha_{2} \gamma_{t h}}{P_{T} \alpha_{3}}+1\right)}-\frac{\alpha_{3} Q}{P_{I} \alpha_{2} \hat{\alpha}_{1} \gamma_{t h}} e^{\frac{\alpha_{3} Q}{P_{I} \alpha_{2} \hat{\alpha}_{1} \gamma_{t h}}} \\
& \Gamma\left(0,\left(\frac{1}{P_{I} \alpha_{2}}+\frac{\gamma_{t h}}{\alpha_{3} P_{T}}\right) \frac{\alpha_{3} Q}{\hat{\alpha}_{1} \gamma_{t h}}\right)
\end{aligned}
$$

which is equivalent to [4, Eq. (15)] with $\sigma=0$, and this also demonstrates the generality of our result.

Note that the closed-form expression for the outage probability with perfect CSI can be obtained by substituting $k_{I}=1$ into (14). 


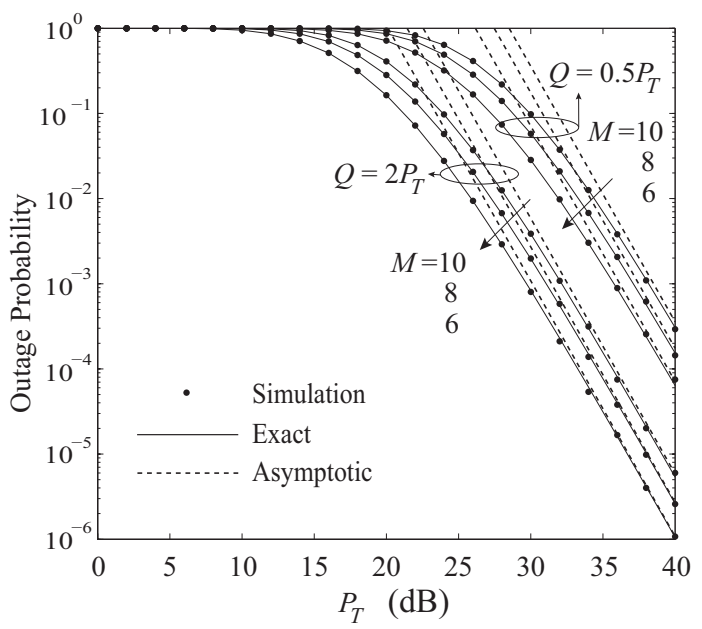

Fig. 1. Outage probability with $L_{c}=2, L=3, P_{0}=10 \%$, and $\rho=0.9$.

\section{B. Asymptotic Analysis}

In this subsection, we characterize the asymptotic behavior of the outage probability in the operating region of high $P_{\mathrm{T}}$. Throughout this subsection, we consider that the SU-Tx power is proportional to the PU-Rx interference. This indicates that the diversity order exists when the PU-Rx is able to tolerate a high amount of interference from the SU-Tx. This will benefit the secondary network without violating the transmission at the primary network. With this in mind, we gather a deeper understanding of the effect of power scaling on the outage probability. Similar to [25], we consider $Q=\mu P_{\mathrm{T}}$, where $\mu$ is positive constant.

In the high-SIR regime with $P_{\mathrm{T}} \rightarrow \infty$, we apply [24, Eq. (3.354.1)] and [24, Eq. (1.211.1)] to derive the first order expansion of $F_{\|\boldsymbol{G}\|^{2}}(x)$ as

$$
F_{\|\boldsymbol{G}\|^{2}}^{\infty}(x) \approx \frac{1}{L_{c}{ }^{L-L_{c}} L_{c} !} x^{L} .
$$

Substituting (17) into (25), the asymptotic outage probability with proportional interference power constraint is presented in the following theorem.

Theorem 2. When $Q=\mu P_{T}$, the asymptotic outage probability of spectrum sharing networks with GSC and multiple $P U$ transceivers as $P_{T} \rightarrow \infty$ is derived as

$$
P_{\text {out }}^{\infty} \approx\left(G_{c} P_{T}\right)^{-G_{d}}
$$

where the diversity order is $G_{d}=L$ and the array gain is

$$
G_{c}=\left[\frac{\Theta}{L_{c}{ }^{L-L_{c}} L_{c} !} \frac{\Gamma(M+L)}{\Gamma(M)}\right]^{-\frac{1}{L}} \frac{1}{\alpha_{2} P_{I} \gamma_{t h}},
$$

with $\Theta=\left(1-e^{-\frac{k_{I} \mu}{\alpha_{1}}}\right)^{M}+\sum_{m=1}^{M}\left(\begin{array}{c}M \\ m\end{array}\right)(-1)^{m+1} \frac{\Gamma\left(L+1, \frac{k_{I} \mu m}{\hat{\alpha}_{1}}\right)}{\left(\alpha_{3} k_{I} \mu m / \hat{\alpha}_{1}\right)^{L}}$.

Similarly, the asymptotic outage probability with perfect CSI can be obtained by substituting $k_{I}=1$ into (18). Based on (18), we confirm that the diversity order is entirely determined by the available number of receive antennas at the SU-Rx $L$,

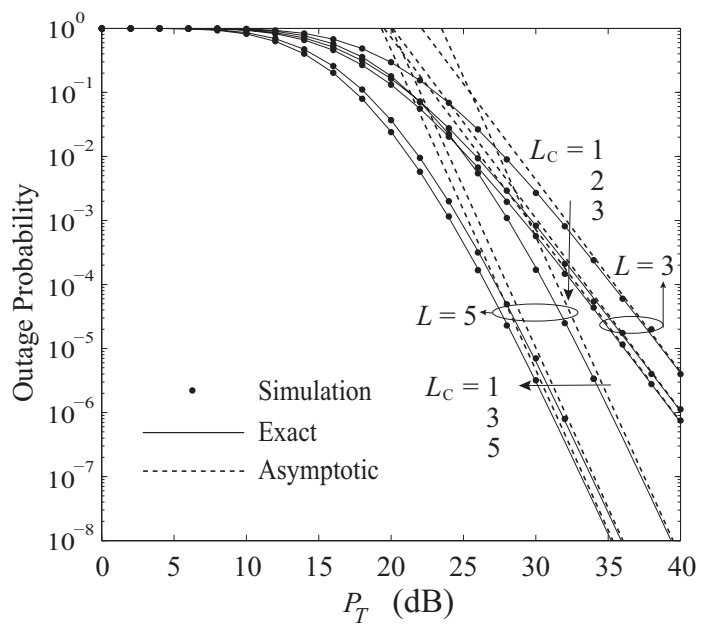

Fig. 2. Outage probability with $M=6, Q=2 P_{\mathrm{T}}, P_{0}=10 \%$, and $\rho=0.9$.

but independent of the number of combined antennas at the SU-Rx $L_{c}$ and the correlation coefficient $\rho$.

We now proceed to examine the outage tradeoff between GSC, MRC, and SC. Given that GSC, MRC, and SC maintain the same diversity order, the tradeoff between them is entirely determined by their respective array gains. Based on our results in (19), we present the following remarks.

Remark 1: We set $L_{c}=L$ in (19) to obtain the array gain of MRC as

$$
G_{c}^{\mathrm{MRC}}=\left[\frac{\Theta \Gamma(M+L)}{L ! \Gamma(M)}\right]^{-\frac{1}{L}} \frac{1}{\alpha_{2} P_{\mathrm{I}} \gamma_{t h}} .
$$

Using (19) and (20), the SNR gap between GSC and MRC is expressed as

$$
\Delta_{1}=10 \log \left(\frac{G_{c}}{G_{c}^{\mathrm{MRC}}}\right)=\left(\frac{10}{L}\right) \log \left(\frac{L_{c}^{L-L_{c}} L_{c} !}{L !}\right) \mathrm{dB} .
$$

Remark 2: We set $L_{c}=1$ in (19) to obtain the array gain of $\mathrm{SC}$ as

$$
G_{c}^{\mathrm{SC}}=\left[\frac{\Theta \Gamma(M+L)}{\Gamma(M)}\right]^{-\frac{1}{L}} \frac{1}{\alpha_{2} P_{\mathrm{I}} \gamma_{t h}} .
$$

Using (19) and (22), the SIR gap between GSC and SC is expressed as

$$
\Delta_{2}=10 \log \left(\frac{G_{c}}{G_{c}^{\mathrm{SC}}}\right)=\left(\frac{10}{L}\right) \log \left(L_{c}^{L-L_{c}} L_{c} !\right) \mathrm{dB} .
$$

Remark 3: Using (20) and (22), the SIR gap between MRC and $\mathrm{SC}$ is expressed as

$$
\Delta_{3}=10 \log \left(\frac{G_{c}^{\mathrm{MRC}}}{G_{c}^{\mathrm{SC}}}\right)=\left(\frac{10}{L}\right) \log (L !) \mathrm{dB} .
$$

Based on Remarks 1, 2, and 3, we confirm that the SIR gaps $\Delta_{1}, \Delta_{2}$, and $\Delta_{3}$ are fully described by two parameters: the number of available receive antennas at the SU-Rx $L$ and the number of combined antennas at the SU-Rx $L_{c}$. 


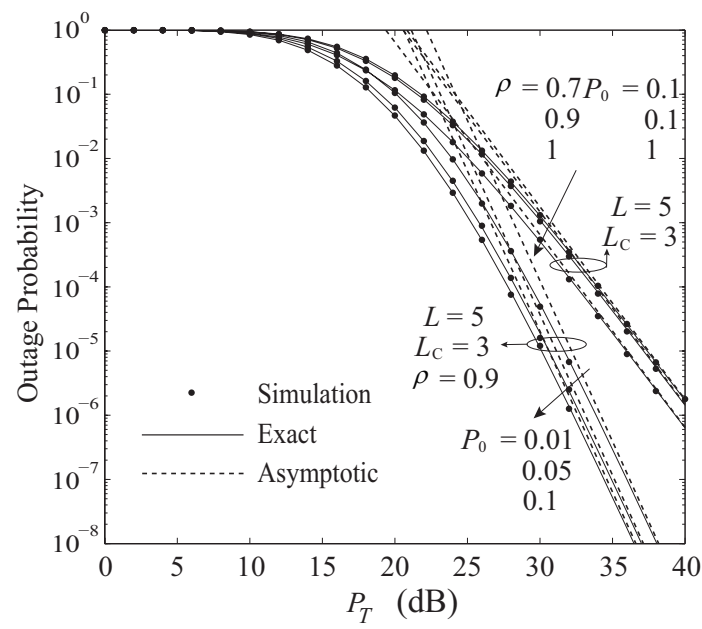

Fig. 3. Outage probability with $L_{c}=3, L=5$, and $M=5$.

\section{Numerical Results}

Numerical examples are provided to highlight the impact of GSC on the performance of underlay spectrum sharing networks with imperfect CSI. In the examples, we assume that the threshold SIR is $\gamma_{\text {th }}=1 \mathrm{~dB}$ and the interference power from PU-Tx is $P_{\mathrm{I}}=3 \mathrm{~dB}$. We also set $\hat{\alpha}_{1}=2, \alpha_{2}=3$, and $\alpha_{3}=1$. We see a perfect match between the simulations and the exact analytical curves (plotted from (14)). We also see that the asymptotic analytical lines (plotted from (18)) are in precise agreement with the exact analytical curves in the medium-to-high regime of $P_{\mathrm{T}}$.

Fig. 1 plots the outage probability versus $P_{\mathrm{T}}$ as we vary $M$. Here, we set $L=3, L_{c}=2, P_{0}=10 \%$, and $\rho=0.9$. We observe that the diversity order is independent of $M$ as indicated by the parallel slopes of the asymptotes. The diversity order is entirely determined by the number of antennas at the SU-Rx as $G_{d}=L$. As expected, the outage probability decreases with decreasing $M$. This is due to the fact that the array gain in (19) increases with decreasing $M$. This result is not surprising, since the interference from the PU-Tx to the SU-Rx increases with increasing $M$, as shown in (5), which negatively impacts the secondary network. We also observe that setting $Q=2 P_{\mathrm{T}}$ achieves a lower outage probability compared to $Q=0.5 P_{\mathrm{T}}$. This is due to the fact that the array gain in (19) increases with increasing $\mu$. In other words, the higher peak interference power constraint at PUs enables more reliable transmission of the secondary network.

Fig. 2 plots the outage probability versus $P_{\mathrm{T}}$ as we vary $L_{c}$. Here, we set $M=6, Q=2 P_{\mathrm{T}}, P_{0}=10 \%$, and $\rho=0.9$. We observe that the diversity order is $G_{d}=L$ regardless of $L_{c}$. Setting $L_{c}=L$ and $L_{c}=1$, we obtain the special cases of MRC and SC, respectively. We also observe that the SIR gap between MRC and SC are accurately predicted according to (24). As expected, we observe that the outage probability decreases with increasing $L_{c}$. This is due to the fact that the array gain increases with increasing $L_{c}$, as shown in (19). Moreover, we find that the lower outage probability can be achieved by employing more antennas $L$, which can

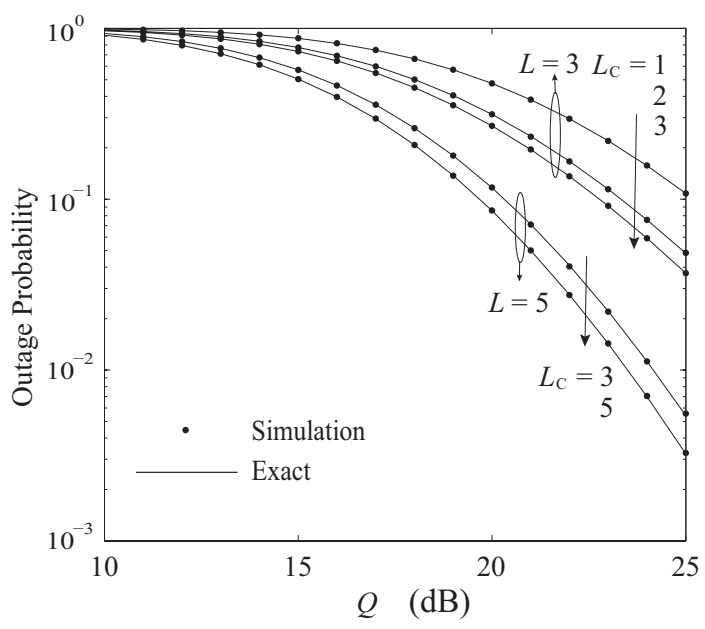

Fig. 4. Outage probability with $M=5, P_{T}=25 \mathrm{~dB}, P_{0}=10 \%$, and $\rho=0.9$.

be explained by the fact that the array gain increases with increasing $L$.

Fig. 3 plots the outage probability versus $P_{\mathrm{T}}$ as we vary $\rho$ and $P_{0}$. Here, we set $M=5, L=5, L_{c}=3$, and $Q=1.5 P_{\mathrm{T}}$. We can easily see the same parallel slopes for different coefficients $\rho$, which indicates that the diversity order is independent of $\rho$ and $P_{0}$. Interestingly, for the case of $P_{0}=0.1$, the outage probability decreases as $\rho$ increases; for the case of $\rho=0.9$, the outage probability decreases with increasing $P_{0}$. This can be explained by the fact that increasing $\rho$ and $P_{0}$ ensure the higher transmit power of the SU-Tx, and thus brings about better outage performance. As expected, for $\rho=1$, which refers to perfect CSI, the lowest outage probability is achieved.

Fig. 4 plots the outage probability versus $Q$ as we vary $L_{c}$. Here, we set $M=5, P_{\mathrm{T}}=25 \mathrm{~dB}, P_{0}=10 \%$, and $\rho=0.9$. As expected, the outage probability decreases with increasing $Q$ when $P_{T}$ is not a linear function of $Q$. Furthermore, the outage probability decreases with increasing $L_{c}$.

\section{CONCLUSIONS}

We proposed generalized selection combining (GSC) in interference-limited spectrum sharing networks with outdated channel state information and multiple PU transceivers. Our aim is to examine the impact of GSC and outdated channel on the outage probability. To facilitate this, we derived new closed-form expressions for the exact and the asymptotic outage probability. The SU-Rx is allowed to combine the $L_{c}$ strongest antennas out of $L$ antennas. With this in mind, we examined the fundamental question of how $L_{c}, M$, and $\rho$ affect the transmit power at SU-Tx, the interference from SUTx to PU-Rx, and the interference from PU-Tx to SU-Rx. The valuable insights are reached that the interference power at the SU-Rx increases as $M$ increases, and the outage probability is improved with more accurate CSI. 


\section{APPENDIX A}

\section{PROOF OF THEOREM 1}

According to (5), the CDF of $\tilde{\gamma}$ conditioned on $V$ is written as

$$
\begin{aligned}
F_{\tilde{\gamma} \mid V}(\gamma)= & \underbrace{\operatorname{Pr}\left\{\|\boldsymbol{G}\|^{2} \leq \frac{\gamma V}{P_{T}},\left|\hat{h}_{1 m^{*}}\right|^{2}<k_{I} \frac{Q}{P_{T}}\right\}}_{I_{1}(V)} \\
& +\underbrace{\operatorname{Pr}\left\{\frac{\|\boldsymbol{G}\|^{2}}{\left|\hat{h}_{1 m^{*}}\right|^{2}} \leq \frac{\gamma V}{k_{I} Q},\left|\hat{h}_{1 m^{*}}\right|^{2} \geq k_{I} \frac{Q}{P_{T}}\right\}}_{I_{2}(V)} .
\end{aligned}
$$

By substituting (7) and (12) into (25), the first term $I_{1}(Z)$ is obtained as

$$
I_{1}(V)=\sum_{S_{k} \in S_{K}} \alpha_{k}\left(\frac{\gamma V}{P_{T}}\right)^{\beta_{k}} e^{-\frac{\delta_{k} \gamma V}{\alpha_{3} P_{T}}}\left(1-e^{-\frac{k_{I} Q}{\alpha_{1} P_{T}}}\right)^{M} .
$$

Integrating (26) with respect to the PDF of $Z$ given by (13) results in

$$
\begin{aligned}
E_{V}\left\{I_{1}(V)\right\}= & \left(1-e^{-\frac{k_{I} Q}{\alpha_{1} P_{T}}}\right)^{M}\left(\frac{1}{P_{I} \alpha_{2}}\right)^{M} \frac{1}{\Gamma(M)} \\
& \sum_{S_{k} \in S_{K}} \alpha_{k}\left(\frac{\gamma}{P_{T}}\right)^{\beta_{k}} \frac{\Gamma\left(\beta_{k}+M\right)}{\left(\frac{\delta_{k} \gamma}{P_{T} \alpha_{3}}+\frac{1}{P_{I} \alpha_{2}}\right)^{\beta_{k}+M} .}
\end{aligned}
$$

Applying the same approach, $E_{V}\left\{I_{2}(V)\right\}$ can be derived with the help of [24, Eq. (8.352.2)], [24, Eq. (9.211.4.8)], and the polynomial expansion, which yields

$$
\begin{aligned}
& E_{V}\left\{I_{2}(V)\right\}=\sum_{m=1}^{M}\left(\begin{array}{c}
M \\
m
\end{array}\right)(-1)^{m+1} \sum_{S_{k} \in S_{K}} \alpha_{k}\left(\frac{\gamma}{k_{I} Q}\right)^{\beta_{k}} \beta_{k} ! \\
& \left(\frac{1}{P_{I} \alpha_{2}}\right)^{M} \frac{\Gamma\left(\beta_{k}+M\right)}{\Gamma(M)} e^{-\frac{m k_{I} Q}{\alpha_{1} P_{T}}} \sum_{i=0}^{\beta_{k}} \frac{\left(\frac{k_{I} Q}{P_{T}}\right)^{i}}{i !}\left(\frac{\alpha_{3} k_{I} Q}{\hat{\alpha}_{1} \delta_{k} \gamma}\right)^{\beta_{k}+M} \\
& \left(\frac{m}{\hat{\alpha}_{1}}\right)^{i+M} \Phi\left(\beta_{k}+M, M+i ;\left(\frac{1}{P_{I} \alpha_{2}}+\frac{\delta_{k} \gamma}{\alpha_{3} P_{T}}\right) \frac{\alpha_{3} m k_{I} Q}{\hat{\alpha}_{1} \delta_{k} \gamma}\right) .
\end{aligned}
$$

Substituting (27) and (28) into (25), the CDF of the SIR is finally derived as (14).

\section{REFERENCES}

[1] A. Goldsmith, S. Jafar, I. Maric, and S. Srinivasa, "Breaking spectrum gridlock with cognitive radios: An information theoretic perspective," Proc. IEEE, vol. 97, no. 5, pp. 894-914, May. 2009.

[2] M. Gastpar, "On capacity under receive and spatial spectrum-sharing constraints," IEEE Trans. Inf. Theory, vol. 53, no. 2, pp. 471-487, Feb. 2007.

[3] R. Zhang, "On peak versus average interference power constraints for protecting primary users in cognitive radio networks," IEEE Trans. Wireless Commun., vol. 8, no. 4, pp. 2112-2120, Apr. 2009.
[4] H. A. Suraweera, P. J. Smith, and M. Shafi, "Capacity limits and performance analysis of cognitive radio with imperfect channel knowledge," IEEE Trans. Veh. Technol., vol. 59, no. 4, pp. 1811-1822, May. 2010.

[5] Y. Zou, Y.-D. Yao, and B. Zheng, "Outage probability analysis of cognitive transmissions: Impact of spectrum sensing overhead," IEEE Trans. Wireless Commun., vol. 9, no. 8, pp. 2676-2688, Aug. 2010.

[6] D. Li, "Performance analysis of MRC diversity for cognitive radio systems," IEEE Trans. Veh. Technol., vol. 61, no. 2, pp. 849-853, Feb. 2012.

[7] Q. Wu, Y. Huang, J. Wang, and Y. Cheng, "Effective capacity of cognitive radio systems with GSC diversity under imperfect channel knowledge," IEEE Commun. Lett., vol. 16, no. 11, pp. 1792-1795, Nov. 2012.

[8] R. Duan, R. Jäntti, M. Elmusrati, and R. Virrankoski, "Capacity for spectrum sharing cognitive radios with MRC diversity and imperfect channel information from primary user," in Proc. IEEE Global Commun. Conf., Miami, USA, Dec. 2010, pp. 1-5.

[9] T. Q. Duong, D. B. da Costa, T. Tsiftsis, C. Zhong, and A. Nallanathan, "Outage and diversity of cognitive relaying systems under spectrum sharing environments in Nakagami-m fading," IEEE Commun. Lett., vol. 16, no. 12, pp. 2075-2078, Dec. 2012.

[10] J.-B. Kim and D. Kim, "Outage probability and achievable diversity order of opportunistic relaying in cognitive secondary radio networks," IEEE Trans. Commun., vol. 60, no. 9, pp. 2456-2466, Sep. 2012.

[11] T. Q. Duong, P. L. Yeoh, V. N. Q. Bao, M. Elkashlan, and N. Yang, "Cognitive relay networks with multiple primary transceivers under spectrum-sharing," IEEE Signal Process. Lett., vol. 19, no. 11, pp. 741744, Nov. 2012

[12] M. Win and J. Winters, "Virtual branch analysis of symbol error probability for hybrid selection/maximal-ratio combining in Rayleigh fading," IEEE Trans. Commun., vol. 49, no. 11, pp. 1926-1934, Nov. 2001.

[13] D. S. Michalopoulos, H. A. Suraweera, G. K. Karagiannidis, and R. Schober, "Amplify-and-Forward relay selection with outdated channel estimates," IEEE Trans. Commun., vol. 60, no. 5, pp. 1278-1290, May. 2012.

[14] Q. Wu, Z. Zhang, and J. Wang, "Outage analysis of cognitive relay networks with relay selection under imperfect CSI environment," IEEE Commun. Lett., vol. 17, no. 7, pp. 1297-1300, Jul. 2013.

[15] H. Kim, H. Wang, S. Lim, and D. Hong, "On the impact of outdated channel information on the capacity of secondary user in spectrum sharing environments," IEEE Trans. Wireless Commun., vol. 11, no. 1, pp. 284-295, Dec. 2012.

[16] A. Ghasemi and E. S. Sousa, "Fundamental limits of spectrum-sharing in fading environments," IEEE Trans. Wireless Commun., vol. 6, no. 2, pp. 649-658, Feb. 2007.

[17] X. Cai and G. B. Giannakis, "Performance analysis of combined transmit selection diversity and receive generalized selection combining in Rayleigh fading channels," IEEE Trans. Wireless Commun., vol. 3, no. 6, pp. 1980-1983, Nov. 2004.

[18] T. L. Singal, Wireless communications. New Delhi: Tata McGraw-Hill Education, 2010.

[19] P. L. Yeoh, M. Elkashlan, K. J. Kim, T. Q. Duong, and G. K. Karagiannidis, "Cognitive MIMO relaying with multiple primary transceivers," Proc. IEEE Global Commun. Conf., Atlanta, GA, Dec. 2013.

[20] S. A. R. Zaidi, M. Ghogho, D. McLernon, and A. Swami, "Achievable spatial throughput in multi-antenna cognitive underlay networks with multi-hop relaying," IEEE J. Sel. Areas Commun., vol. 31, no. 8, pp. 1278-1290, Aug. 2013

[21] K. J. Kim, T. Q. Duong, H. V. Poor, and L. Shu, "Performance analysis of cyclic prefixed single-carrier spectrum sharing relay systems in primary user interference," IEEE Trans. Signal Process., vol. 60, no. 12 pp. 6729-6734, Dec. 2012

[22] R. L. Graham, D. E. Knuth, and O. Patashnik, Concrete Mathematics. New York: Addison-Wesley, 1989.

[23] H. Q. Ngo, M. Matthaiou, T. Q. Duong, and E. G. Larsson, "Uplink performance analysis of multicell MU-MIMO systems with ZF receivers," IEEE Trans. Veh. Technol., vol. 62, no. 9, pp. 4471-4482, Nov. 2013.

[24] I. S. Gradshteyn and I. M. Ryzhik, Table of Integrals, Series and Products, 6th ed. New York, NY, USA: Academic Press, 2000.

[25] T. Q. Duong, D. B. da Costa, M. Elkashlan, and V. N. Q. Bao, "Cognitive amplify-and-forward relay networks over Nakagami-m fading," IEEE Trans. Veh. Technol., vol. 61, no. 5, pp. 2368-2374, Dec. 2012. 


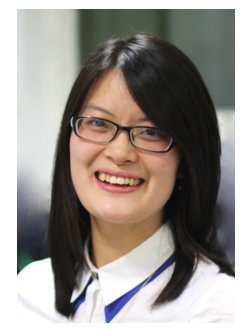

Yansha Deng (S'13) is working towards her Ph.D. degree in Electronic Engineering at Queen Mary University of London. Since Fall 2010, she has been working towards the M.S. degree in Central South University. Her research interests include multipleantenna systems, cooperative networks, molecular communication, and physical layer security.

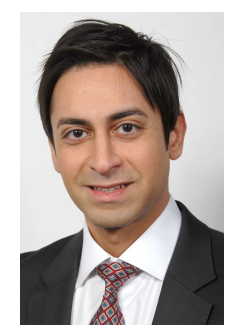

Maged Elkashlan (M'06) received the Ph.D. degree in Electrical Engineering from the University of British Columbia, Canada, 2006. From 2006 to 2007, he was with the Laboratory for Advanced Networking at University of British Columbia. From 2007 to 2011, he was with the Wireless and Networking Technologies Laboratory at Commonwealth Scientific and Industrial Research Organization (CSIRO), Australia. During this time, he held an adjunct appointment at University of Technology Sydney, Australia. In 2011, he joined the School of Electronic Engineering and Computer Science at Queen Mary University of London, UK, as an Assistant Professor. He also holds visiting faculty appointments at the University of New South Wales, Australia, and Beijing University of Posts and Telecommunications, China. His research interests fall into the broad areas of communication theory, wireless communications, and statistical signal processing for distributed data processing, millimeter wave communications, cognitive radio, and wireless security.

Dr. Elkashlan currently serves as an Editor of the IEEE TRANSACTIONS ON Wireless COMMUNiCATIONS, the IEEE TRANSACTIONS ON Vehicular TECHNOLOGY, and the IEEE COMMUNICATIONS LETTERS. He also serves as the Lead Guest Editor for the special issue on "Green Media: The Future of Wireless Multimedia Networks" of the IEEE WIRELESS COMMUNICATIONS MAGAZINE, Lead Guest Editor for the special issue on "Millimeter Wave Communications for 5G" of the IEEE Communications MaGazine, Guest Editor for the special issue on "Energy Harvesting Communications" of the IEEE COMMUNICATIONS MAGAZINE, and Guest Editor for the special issue on "Location Awareness for Radios and Networks" of the IEEE JOURNAL on Selected Areas in Communications. He received the Best Paper Award at the IEEE Vehicular Technology Conference (VTC-Spring) in 2013. He received the Exemplary Reviewer Certificate of the IEEE Communications Letters in 2012.

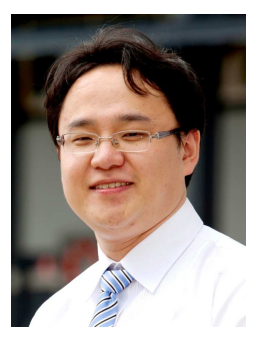

Nan Yang (S'09-M'11) received the B.S. degree in electronics from China Agricultural University, Beijing, China, in 2005, and the M.S. and Ph.D. degrees in electronic engineering from the Beijing Institute of Technology in 2007 and 2011, respectively. From 2008 to 2010, he was a Visiting Ph.D. Student with the School of Electrical Engineering and Telecommunications, University of New South Wales, Sydney, Australia. From 2010 to 2012, he was a Post-Doctoral Research Fellow with the Wireless and Networking Technologies Laboratory, Commonwealth Scientific and Industrial Research Organization, Marsfield, Australia. Since 2012, he has been with the School of Electrical Engineering and Telecommunications, University of New South Wales, where he is currently a Post-Doctoral Research Fellow. His general research interests include communications theory and signal processing, with specific interests in collaborative networks, multiple-antenna systems, network security, and distributed data processing.

Dr. Yang received the Exemplary Reviewer Certificate of the IEEE COMMUNICATIONS LETTERS in 2012 and 2013 and the Best Paper Award at the IEEE 77th Vehicular Technology Conference (VTC-Spring) in 2013. He is currently serving as the Editor of the Transactions on Emerging Telecommunications Technologies. He has served as Technical Program Committee Member for several IEEE conferences, such as the IEEE International Conference on Communications and the IEEE Global Communications Conference. 


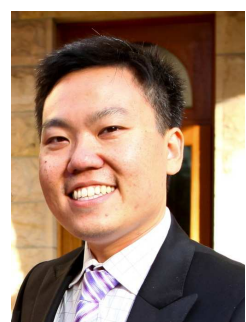

Phee Lep Yeoh (S'08, M'12) received the B.E. degree with University Medal from the University of Sydney, Australia, in 2004, and the Ph.D. degree from the University of Sydney, Australia, in 2012. From 2008 to 2012, he was with the Telecommunications Laboratory at the University of Sydney and the Wireless and Networking Technologies Laboratory at the Commonwealth Scientific and Industrial Research Organization (CSIRO), Australia. In 2012, he joined the Department of Electrical and Electronic Engineering at the University of Melbourne, Australia. He is a recipient of the Australian Research Council Discovery Early Career Researcher Award, University of Sydney Postgraduate Award, and CSIRO Postgraduate Scholarship. His research interests include heterogeneous networks, large-scale MIMO, cooperative communications, and cognitive networks.

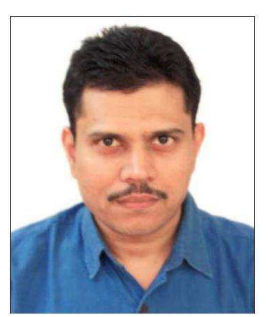

Ranjan K. Mallik (S'88-M'93-SM'02-F'12) received the B.Tech. degree from the Indian Institute of Technology, Kanpur, in 1987 and the M.S. and $\mathrm{Ph} . \mathrm{D}$. degrees from the University of Southern California, Los Angeles, in 1988 and 1992, respectively, all in Electrical Engineering. From August 1992 to November 1994, he was a scientist at the Defence Electronics Research Laboratory, Hyderabad, India, working on missile and EW projects. From November 1994 to January 1996, he was a faculty member of the Department of Electronics and Electrical Communication Engineering, Indian Institute of Technology, Kharagpur. From January 1996 to December 1998, he was with the faculty of the Department of Electronics and Communication Engineering, Indian Institute of Technology, Guwahati. Since December 1998, he has been with the faculty of the Department of Electrical Engineering, Indian Institute of Technology, Delhi, where he is currently a Professor. His research interests are in diversity combining and channel modeling for wireless communications, space-time systems, cooperative communications, multiple-access systems, power line communications, difference equations, and linear algebra.

Dr. Mallik is a member of Eta Kappa Nu. He is also a member of the IEEE Communications, Information Theory, and Vehicular Technology Societies, the American Mathematical Society, and the International Linear Algebra Society, a fellow of IEEE, the Indian National Academy of Engineering, the Indian National Science Academy, The National Academy of Sciences, India, Allahabad, the Indian Academy of Sciences, Bangalore, The World Academy of Sciences - for the advancement of science in developing countries (TWAS), The Institution of Engineering and Technology, U.K., and The Institution of Electronics and Telecommunication Engineers, India, a life member of the Indian Society for Technical Education, and an associate member of The Institution of Engineers (India). He is an Area Editor for the IEEE TRANSACTIONS ON WIRELESS COMMUNICATIONS. He is a recipient of the Hari Om Ashram Prerit Dr. Vikram Sarabhai Research Award in the field of Electronics, Telematics, Informatics, and Automation, and of the Shanti Swarup Bhatnagar Prize in Engineering Sciences. 